\title{
APPLICATIONS OF MARTINGALE SYSTEM THEOREMS
}

\author{
BY \\ J. L. SNELL(1)
}

1. Basic definitions. The following definitions are all relative to a triple $(\Omega, \mathcal{B}, \operatorname{Pr})$ in which $\Omega$ is a space with points denoted by $\omega, \mathcal{B}$ is a Borel field of subsets of $\Omega$ which includes $\Omega$, and $\operatorname{Pr}$ is a completely additive set function defined on sets of $B$ such that for $\Lambda \in \mathcal{B}, 0 \leqq \operatorname{Pr}\{\Lambda\} \leqq \operatorname{Pr}\{\Omega\}=1$. A random variable is a function defined on $\Omega$ having values on the real line extended by the adjunction of $-\infty,+\infty$ and which is measurable with respect to $B$, i.e., for every real number $r$, the $\omega$ set $\{x(\omega) \leqq r\}$ is an element of $\mathcal{B}$. In the following the Borel fields discussed will be assumed to be subfields of $\mathbb{B}$ which include the set $\Omega$. If $\left\{x_{t}, t \in T\right\}$ is any collection of random variables, the Borel field generated by this collection is the smallest Borel field with respect to which every member of the collection is measurable. A stochastic process is a collection $\left\{x_{t}, F_{t}, t \in T\right\}$, where $T$ is a subset of the extended real line, $\left\{F_{t}, t \in T\right\}$ is an increasing collection of Borel fields in the sense that for $t_{1}<t_{2}, F_{t_{1}} \subset F_{t_{2}}$, and $x_{t}$ is a random variable measurable with respect to $F_{t}$ or equal for almost all $\omega$ to such a function. If the collection $\left\{x_{t}, t \in T\right\}$ is referred to as a stochastic process, it will be understood that $F_{t}$ is the Borel field generated by $\left\{x_{s}, s \leqq t, s \in T\right\}$.

By means of the probability measure Pr we define a Lebesgue integral and if a random variable $x$ is integrable, we say that the expected value of $x$ exists and define the expected value, written $E\{x\}$, by $E\{x\}=\int_{\Omega} x d p r$. We do not require that $E\{x\}$ be finite. Let $x$ be a random variable such that $E\{x\}$ exists and $F$ be a Borel field. Then $\phi$ defined by $\phi\{\Lambda\}=\int_{\Lambda} x d p r$ for $\Lambda \in F$ is a completely additive set function defined on $F$ which is absolutely continuous with respect to probability measure, in the sense that $\phi\{\Lambda\}=0$ if $\operatorname{Pr}\{\Lambda\}=0$. By a generalization of the Radon-Nikodým theorem $[5$, p. 169] there exists a random variable $y$ which is measurable with respect to $F$, for which $E\{y\}$ exists, and such that $\phi\{\Lambda\}=\int_{\Lambda} y d p r$. The random variable $y$ is unique up to a set of probability measure zero. This discussion justifies the following definition.

DEFINITION 1.1. Let $x$ be a random variable such that $E\{x\}$ exists and let $F$ be a Borel field. The conditional expectation of $x$ relative to $F$, written $E\{x \| F\}$, is defined as any $\omega$ function $y$ which is equal for almost all $\omega$ to a

Presented to the Society September 6, 1951; received by the editors November 21, 1951.

(1) This work was done in part in connection with an ONR project at Cornell University. The results were submitted in a thesis in partial fulfillment of the requirements for the degree of Doctor of Philosophy at the University of Illinois. The thesis was written under the direction of Professor J. L. Doob. 
function measurable with respect to $F$, such that $E\{y\}$ exists, and such that

$$
\int_{\Lambda} y d p r=\int_{\Lambda} x d p r
$$

for all $\Lambda \in F$.

Definition 1.2. A stochastic process $\left\{x_{t}, F_{t}, t \in T\right\}$ is a martingale if

$$
\begin{aligned}
& E\left\{\left|x_{t}\right|\right\}<\infty \\
& E\left\{x_{t} \| F_{s}\right\}=x_{s}
\end{aligned}
$$$$
\text { for all } t \in T
$$

with probability one whenever $s<t$.

Such a stochastic process is a semimartingale if condition (a) is satisfied and condition (b) is replaced by

$$
E\left\{x_{t} \| F_{s}\right\} \geqq x_{s}
$$

with probability one whenever $s<t$.

If in the above definitions condition (a) is weakened to require only that $E\left\{x_{t}\right\}$ exists as a finite or infinite number, we shall refer to tnese processes as generalized martingales and generalized semimartingales.

The development of martingales and semimartingales is contained in a forthcoming book by J. L. Doob [3, Chapter 7]. We shall give here some of the basic properties of these processes. The following two theorems are due to Doob.

TheоRем 1.1. If $\left\{x_{n}, F_{n}, n \geqq 1\right\}$ is a semimartingale such that $\sup _{n} E\left\{\left|x_{n}\right|\right\}$ $<\infty$, then $\lim _{n \rightarrow \infty} x_{n}=x_{\infty}$ exists with probability one, and $E\left\{\left|x_{\infty}\right|\right\}<\infty$.

THEOREM 1.2. Let $z$ be a random variable with $E\{|z|\}<\infty$ and $F_{1} \subset F_{2}$ $\subset$.. be an increasing sequence of Borel fields. Then $E\left\{z \| F_{n}\right\}, F_{n}, 1 \leqq n \leqq \infty$, is a martingale and $\lim _{n \rightarrow \infty} E\left\{z \| F_{n}\right\}=E\left\{z \| F_{\infty}\right\}$. Here $F_{\infty}$ is the smallest Borel field such that $F_{\infty} \supset \cup F_{n}$. The random variables of this martingale are uniformly integrable.

Note that by condition (b) of Definition 1.2 , if $\left\{x_{n}, F_{n}, n \geqq 1\right\}$ is a martingale, then $E\left\{x_{n}\right\}=E\left\{x_{1}\right\}$ for all $n$. Hence Theorem 1.1 implies that any martingale with non-negative random variables converges with probability one. If $\left\{x_{n}, F_{n}, n \geqq 1\right\}$ is a semimartingale, then by condition ( $\left.\mathrm{b}^{\prime}\right)$ we have that $E\left\{x_{n}\right\}$ is monotone nondecreasing with $n$, so that if the random variables are nonpositive, Theorem 1.2 implies that the random variables converge with probability one.

The following interpretation of the elements of a stochastic process has been found to be useful in the study of martingales. Let $\left\{x_{n}, F_{n}, n \geqq 1\right\}$ be a stochastic process. Consider $x_{1}$ to be a gambler's initial fortune and $x_{n}$ his fortune after $n-1$ plays in a gambling game. Let $F_{n}$ represent his knowledge of the past and present after $n-1$ plays. This knowledge includes the values 
of the first $n$ random variables of the process. If $\left\{x_{n}, F_{n}, n \geqq 1\right\}$ is a generalized semimartingale, the property $E\left\{x_{n+1} \| F_{n}\right\} \geqq x_{n}$ can be interpreted to mean that the game is favorable to the gambler. In the case of a generalized martingale, $E\left\{x_{n+1} \| F_{n}\right\}=x_{n}$ can be interpreted to mean that the game is fair. Such an interpretation has suggested a class of theorems called system theorems. These theorems are suggested by the fact that a favorable or a fair game should still be favorable or fair if the gambler adopts a system of play in place of simply making every play. For example suppose that the gambler is permitted at each stage of play either to make the $n$th play with resulting gain $x_{n+1}-x_{n}$ or to pass up the play with resulting zero gain. A system of play for the gambler then can be described by a sequence $\left\{u_{n}\right\}$ of random variables such that $u_{n}(\omega)=1$ if the gambler decides to make the $n$th play and $u_{n}(\omega)=0$ if the gambler decides not to make this play. We impose the condition $\left\{u_{n}(\omega)=1\right\} \in F_{n}$ corresponding to the fact that the player must base his decision whether or not to make the $n$th play only on his knowledge of the past and present. Under such a system of play his fortune is given by $\left\{\bar{x}_{n}\right\}$ where $\bar{x}_{n}$ is defined by

$$
\bar{x}_{n}=x_{1}+\sum_{1}^{n-1} u_{j}\left(x_{i+1}-x_{j}\right) \text {. }
$$

The gambling interpretation suggests that if $\left\{x_{n}, F_{n}, n \geqq 1\right\}$ is a semimartingale (martingale), then the stochastic process $\left\{\bar{x}_{n}, F_{n}, n \geqq 1\right\}$ should also be a semimartingale (martingale). Also in the case of the semimartingale we should expect that $E\left\{\bar{x}_{n}\right\} \leqq E\left\{x_{n}\right\}$ corresponding to the intuitive idea that, the game being favorable, there should be no advantage to omitting any of the plays. In $\$ 2$ of this paper we shall give a "system" theorem suggested by the above reasoning, and show the application of such a theorem to the study of convergence properties of semimartingales. In $\$ 3$ we shall discuss a game problem which has applications in statistical decision theory.

2. Convergence properties of generalized semimartingales. The following system theorem is suggested by the discussion at the end of $\$ 1$. A closely related theorem has been studied by Halmos [6].

Theorem 2.1. Let $X=\left\{x_{n}, F_{n}, n \geqq 1\right\}$ be a martingale and $\left\{u_{n}, n \geqq 1\right\} a$ sequence of random variables such that $u_{n}$ is measurable with respect to $F_{n}$. Define $\bar{x}_{n}$ by

$$
\tilde{x}_{n}=\sum_{1}^{n-1} u_{j}\left(x_{j+1}-x_{j}\right)+x_{1} .
$$

If $E\left\{\left|\bar{x}_{n}\right|\right\}<\infty$ for all $n$, then $\bar{X}=\left\{\bar{x}_{n}, F_{n}, n \geqq 1\right\}$ is a martingale. If $X$ is a semimartingale and the $u_{n}$ 's are non-negative, then $\bar{X}$ is a semimartingale. If in this case the $u_{n}$ 's take on only the values 0 and 1 , we have

$$
E\left\{\bar{x}_{n} \| F_{1}\right\} \leqq E\left\{x_{n} \| F_{1}\right\}
$$


with probability one.

Proof. From the definition of $\bar{x}_{n}$ we have

$$
E\left\{\bar{x}_{n+1}-\bar{x}_{n} \| F_{n}\right\}=E\left\{u_{n}\left(x_{n+1}-x_{n}\right) \| F_{n}\right\}
$$

with probability one. Since $u_{n}$ is measurable with respect to $F_{n}$,

$$
E\left\{u_{n}\left(x_{n+1}-x_{n}\right)|| F_{n}\right\}=u_{n} E\left\{x_{n+1}-x_{n} \| F_{n}\right\}
$$

with probability one. Hence if $X$ is a martingale, the right side of (2.1.2) is equal to 0 , and if $X$ is a semimartingale and $u_{n} \geqq 0$, the right side of (2.1.2) is greater than or equal to 0 . Hence, by (2.1.1), $\bar{X}$ is a martingale in the first case and a semimartingale in the second. Finally, assume that $u_{n}$ takes on only the values 0 or 1 . Let $V \in F_{1}$. Then,

$$
\int_{V}\left(x_{n}-\bar{x}_{n}\right) d p r=\sum_{j=1}^{n-1} \int_{\left\{u_{j}(\omega)=0\right\}}\left(x_{j+1}-x_{j}\right) d p r
$$

and since $\left\{u_{j}(\omega)=0\right\} \in F_{j}$ and $X$ is a semimartingale, each term of the sum on the right side of (2.1.3) is greater than or equal to 0 . This completes the proof.

Let $b_{1}, b_{2}, \cdots, b_{n}$ be any real numbers, and let $r_{1}, r_{2}$ be real numbers with $r_{1}<r_{2}$. The number of upcrossings $b$ of the interval $\left[r_{1}, r_{2}\right]$ by $b_{1}, \cdots, b_{n}$ is defined as the number of times the sequence $b_{1}, b_{2}, \cdots, b_{n}$ passes from below $r_{1}$ to above $r_{2}$. More precisely, let $b_{s_{1}}$ be the first $b_{i}$ (if any) for which $b_{i} \leqq r_{1}$, and in general let $b_{s_{j}}$ be the first $b_{i}$ (if any) after $b_{s_{j-1}}$ for which

$$
b_{i} \geqq r_{2} \text { ( } j \text { even), } \quad b_{i} \leqq r_{1}(j \text { odd }) .
$$

Then the number of upcrossings is $b$ where $2 b$ is the largest even integer $j$ for which $b_{s_{j}}$ is defined, and $b=0$ if $b_{s_{2}}$ is not defined.

Doob [4] has proved that if $\left\{x_{n}, F_{n}, 1 \leqq n \leqq m\right\}$ is a martingale, and if $b(\omega)$ is the number of upcrossings of $\left[r_{1}, r_{2}\right]$ by $x_{1}(\omega), \cdots, x_{m}(\omega)$, then

$$
E\{b\} \leqq \frac{E\left\{\left|x_{m}\right|\right\}+\left|r_{1}\right|}{r_{2}-r_{1}} .
$$

We shall now prove a similar inequality for generalized semimartingales.

THEOREM 2.2. Let $\left\{x_{n}, F_{n}, 1 \leqq n \leqq m\right\}$ be a generalized semimartingale, and let $b(\omega)$ be the number of upcrossings of $\left[r_{1}, r_{2}\right]$ by the sequence $x_{1}(\omega), \cdots$, $x_{m}(\omega)$. Then

$$
E\left\{b \| F_{1}\right\} \leqq \frac{E\left\{\left(x_{m}-r_{1}\right)+\| F_{1}\right\}}{r_{2}-r_{1}}
$$

with probability one( $\left.{ }^{2}\right)$.

(2) Throughout this paper we shall use the notation $x^{+}$for $\max (x, 0)$ and $x^{-}$for $\min (x, 0)$. 
Before proving the theorem, we note that if expectations of both sides of (2.2) are taken, we obtain

$$
E\{b\} \leqq \frac{E\left\{\left(x_{m}-r_{1}\right)^{+}\right\}}{r_{2}-r_{1}} \leqq \frac{E\left\{\left|x_{m}\right|\right\}+\left|r_{1}\right|}{r_{2}-r_{1}} .
$$

Thus Doob's result is true also for semimartingales. To prove the theorem we assume first that the $x_{n}$ 's are non-negative and that $r_{1}=0$. Define $\omega$ functions $s_{1}, \cdots, s_{m}$ as in the definition of upcrossings in terms of $x_{1}(\omega)=b_{1}, \cdots$, $x_{m}(\omega)=b_{m}$. Define $s_{n}(\omega)=m+1$ if not otherwise defined. Define random variables $u_{1}, \cdots, u_{m-1}$ by

$$
\begin{aligned}
& u_{n}(\omega)=1 \text { if } s_{i}(\omega) \leqq n<s_{i+1}(\omega) \text { for } i \text { odd } \\
& =0 \text { otherwise. }
\end{aligned}
$$

Then $u_{n}$ is measurable with respect to $F_{n}$ since

$$
\left\{u_{n}(\omega)=1\right\}=\bigcup_{i \text { odd }}\left\{\left\{s_{i}(\omega) \leqq n\right\}-\left\{s_{i+1}(\omega) \leqq n\right\}\right\} .
$$

If we now interpret $x_{1}$ as a gambler's initial fortune and $x_{n}$ as his fortune after $n-1$ plays of a game of chance, then we see that during the plays which occur between $s_{i}(\omega)$ and $s_{i+1}(\omega)$, for $i$ odd and $s_{i+1}(\omega) \leqq m$, the gambler's fortune increases by at least an amount $r_{2}$. Since the number of such intervals is $b(\omega)$, we see that if the gambler were to make only these plays, his final fortune, call it $x(\omega)$, would satisfy the following inequality:

$$
x(\omega) \leqq r_{2} b(\omega) .
$$

Such a system of play is described by means of the $u_{n}$ 's and then $x(\omega)=x_{1}(\omega)$ $+\sum_{j=1}^{n-1} u_{j}(\omega)\left(x_{j+1}(\omega)-x_{j}(\omega)\right)$. If $E\left\{\left|x_{n}\right|\right\}<\infty$ for all $n$, by Theorem 2.1 we have

$$
E\left\{x|| F_{1}\right\} \leqq E\left\{x_{m} \| F_{1}\right\}
$$

with probability one. Without assuming the $E\left\{\left|x_{n}\right|\right\}<\infty$ the proof follows that of Theorem 2.1 since all the relevant expectations exist in this case. Combining (2.2.1) and (2.2.2) we have

$$
E\left\{b \| F_{1}\right\} \leqq \frac{E\left\{x_{m} \| F_{1}\right\}}{r_{2}}
$$

with probability one as was to be proved. To prove the general case in which it is not assi med that the $x_{n}$ 's are non-negative and $r_{1}=0$, we proceed as follows. Let

$$
\bar{x}_{n}=\left(x_{n}-r_{1}\right)^{+}, \quad n=1, \cdots, m .
$$

Then it is easily seen that $\left\{\bar{x}_{n}, F_{n}, 1 \leqq n \leqq m\right\}$ is a generalized semimartingale and that the number of upcrossings of $\left[r_{1}, r_{2}\right]$ by $x_{1}(\omega), \cdots, x_{m}(\omega)$ is the 
same as the number of upcrossings of $\left[0, r_{2}-r_{1}\right]$ by $\bar{x}_{1}(\omega), \cdots, \bar{x}_{m}(\omega)$. Thus by the first part of the proof we have

$$
E\left\{b \| F_{1}\right\} \leqq \frac{E\left\{\bar{x}_{m} \| F_{1}\right\}}{r_{2}-r_{1}}=\frac{E\left\{\left(x_{m}-r_{1}\right)+\| F_{1}\right\}}{r_{2}-r_{1}}
$$

with probability one. This completes the proof.

COROLLARY 2.2. Let $\left\{x_{n}, F_{n}, 1 \leqq n \leqq m\right\}$ be a stochastic process such that the random variables $x_{n}$ are non-negative and such that $\left\{-x_{n}, F_{n}, 1 \leqq n \leqq m\right\}$ is a generalized semimartingale. Let $b(\omega)$ be the number of upcrossings of $\left[r_{1}, r_{2}\right]$ by $x_{1}(\omega), \cdots, x_{m}(\omega)$ where $r_{2}>r_{1}>0$. Then

$$
E\left\{b \| F_{1}\right\} \leqq \frac{2 r_{2}-r_{1}}{r_{2}-r_{1}}
$$

with probability one. .

Proof. Let $b$ be the number of upcrossings of $\left\{-x_{n}, 1 \leqq n \leqq m\right\}$ for the interval $\left[-r_{2},-r_{1}\right]$. Then by Theorem 2.2 we have

$$
E\left\{b|| F_{1}\right\} \leqq \frac{E\left\{\left(-x_{m}+r_{2}\right)+\| F_{1}\right\}}{r_{2}-r_{1}}
$$

with probability one. Since $x_{m}$ is non-negative we have

$$
E\left\{b|| F_{1}\right\} \leqq \frac{r_{2}}{r_{2}-r_{1}}
$$

with probability one. From the definition of $b$

$$
b(\omega) \leqq b(\omega)+1 .
$$

Combining (2.2.3) and (2.2.4) we have

$$
E\left\{b \| F_{1}\right\} \leqq \frac{2 r_{2}-r_{1}}{r_{2}-r_{1}}
$$

with probability one, as was to be proved.

Using Theorem 2.2 it is easy to prove Doob's Theorem 1.1. We shall instead prove a slight generalization of Theorem 1.1 which will apply to generalized semimartingales.

THEOREM 2.3. Let $X=\left\{x_{n}, F_{n}, n \geqq 1\right\}$ be a generalized semimartingale. Then $\lim _{n \rightarrow \infty} x_{n}(\omega)=x_{\infty}(\omega)$ exists as a finite or negatively infinite limit for almost all $\omega$ such that $\inf _{k} \sup _{n} E\left\{x_{n}^{+} \| F_{k}\right\}<\infty$.

We shall prove the theorem by showing that for almost all $\omega$ such that the sequence $\left\{x_{n}(\omega)\right\}$ diverges or converges to $+\infty$ we have for all $k$, with 
probability one,

$$
\sup _{n} E\left\{x_{n}^{+} \| F_{k}\right\}=\infty \text {. }
$$

Let $r_{2}>r_{1}$ be real numbers and define $V_{r_{1} r_{2}}$ by

$$
V_{r_{1} r_{2}}=\left\{\limsup _{n} x_{n}(\omega)>r_{2}>r_{1}>\lim _{n} \inf x_{n}(\omega)\right\} \text {. }
$$

Let $b_{k, m}(\omega)$ be the number of upcrossings of $\left[r_{1}, r_{2}\right]$ by $x_{k}(\omega), \cdots, x_{m}(\omega)$. For $\omega \in V_{r_{1} r_{2}}, b_{k, m}(\omega)$ goes monotonically to $+\infty$ as $n$ approaches infinity. Hence by Theorem 2.2 we have $(2.3 .1)$ for almost all $\omega$ in $V_{r_{1} r_{2}}$. Since any point of divergence is in some $V_{r_{1} r_{2}}$ for $r_{1}$ and $r_{2}$ rational, we have (2.3.1) for almost all points of divergence. Let $\Lambda=\left\{\lim x_{n}(\omega)=+\infty\right\}$. If (2.3.1) is not true for almost all $\omega$ of $\Lambda$, then for some $N$ and some $k_{0}$ the set $\Lambda^{N}=\left\{\sup E\left\{x_{n}^{+} \| F_{k_{0}}\right\}<N\right\}$ is such that $\operatorname{Pr}\left\{\Lambda \Lambda^{N}\right\}>0$. But

$$
\int_{\Lambda^{N}} E\left\{x_{n}^{+} \| F_{k_{0}}\right\} d p r=\int_{\Lambda^{N}} x_{n}^{+} d p r, \quad n \geqq k_{0} .
$$

By Fatou's lemma the right side of (2.3.2) approaches $+\infty$ as $n$ approaches $\infty$. The left side on the other hand is always less than $N \operatorname{Pr}\left\{\Lambda^{N}\right\}$. This is a contradiction. Hence a set such as $\Lambda^{N}$ cannot exist. This completes the proof.

COROLLARY 2.3. Let $\left\{x_{n}, F_{n}, n \geqq 1\right\}$ be a generalized martingale such that for some random variable $z$ with $E\{|z|\}<\infty$, for all $n \geqq 1, E\left\{z \| F_{n}\right\} \leqq x_{n}$ with probability one. Then $\lim _{n \rightarrow \infty} x_{n}(\omega)=x_{\infty}(\omega)$ exists with probability one, is finite for almost all $\omega$ of the set $\left\{\inf _{n} x_{n}(\omega)<\infty\right\}$, and $E\left\{x_{\infty} \| F_{1}\right\} \leqq x_{1}$ with probability one.

Proof. Let $y_{n}=x_{n}-E\left\{z \| F_{n}\right\}$. Then $\left\{y_{n}, F_{n}, n \geqq 1\right\}$ is a generalized martingale with non-negative random variables. By Theorem 1.2

$$
\lim _{n \rightarrow \infty} E\left\{z \| F_{n}\right\}=E\left\{z \| F_{\infty}\right\}
$$

with probability one. By Theorem 2.3, $\lim _{n \rightarrow \infty} y_{n}=y_{\infty}$ exists and is finite for almost all $\omega$ of the set $\left\{\inf _{n} y_{n}(\omega)<\infty\right\}$. Since $\inf _{n} E\left\{z \| F_{n}\right\}=E\left\{z \| F_{\infty}\right\}<\infty$ with probability one, we have that $\lim _{n \rightarrow \infty} x_{n}=x_{\infty}$ exists and is finite for almost all $\omega$ of the set $\left\{\inf x_{n}(\omega)<\infty\right\}$, as was to be proved. Since $y_{n} \geqq 0$ for all $n$ with probability one, it follows from Fatou's lemma that $E\left\{y_{\infty} \| F_{1}\right\} \leqq y_{1}$ with probability one and hence also that $E\left\{x_{\infty} \| F_{1}\right\} \leqq x_{1}$ with probability one.

Let $x$ be a random variable with $E\{|x|\}<\infty$ and $F_{1} \subset F_{2} \subset \cdots$ an increasing sequence of Borel fields. Then by Theorem 1.2, $\lim E\left\{x \| F_{n}\right\}$ $=E\left\{x \| F_{\infty}\right\}$ with probability one, where $F_{\infty}$ is the smallest Borel field which includes $U_{n} F_{n}$. We shall now give an example to show that this result need not hold if it is assumed only that $E\{x\}$ exists.

Consider the probability space $(\Omega, \mathbb{B}, \operatorname{Pr})$ with $\Omega$ the interval $[0,1]$ of the 
real line, $\mathcal{B}$ the set of Lebesgue measurable sets, and $\operatorname{Pr}$ measure as Lebesgue measure. Let $F_{n}$ be the field generated by the intervals $\left[j / 2^{n},(j+1) / 2^{n}\right]$, $j=0,1, \cdots, 2^{n-1}$. Let $F_{\infty}$ be the smallest Borel field containing $\bigcup_{n} F_{n}$. Define measurable functions $\left\{x_{n}, n \geqq 1\right\}$ such that $x_{n}$ is non-negative, and such that

$$
\operatorname{Pr}\left\{x_{n}(\omega) \leqq \frac{1}{2^{n}}\right\} \geqq 1-\frac{1}{2^{n}}
$$

and

$$
\int_{\left[j / 2^{n},(j+1) / 2^{n}\right]} x_{n} d p r \geqq \frac{1}{n}, \quad j=0,1, \cdots, 2^{n-1} .
$$

Let $x=\sum x_{n}$. Then $x$ is finite-valued with probability one and

$$
\begin{aligned}
\int_{\left[j / 2^{n},(j+1) / 2^{n^{n}}\right]} x d p r & =\sum \int_{\left[j / 2^{n},(j+1) / 2^{n}\right]} x_{n} d p r \\
& \geqq \sum_{n} \frac{1}{n} .
\end{aligned}
$$

Therefore, if $V \in F_{n}, \int_{V} x d p r=\infty$, so that $E\left\{x \| F_{n}\right\}=\infty$ with probability one. On the other hand $E\left\{x \| F_{\infty}\right\}=x$ with probability one since $x$ is measurable with respect to $F_{\infty}$. However, $x$ is finite with probability one, so that $E\left\{x \| F_{\infty}\right\}<\lim _{n \rightarrow \infty} E\left\{x \| F_{n}\right\}$ with probability one.

THEOREM 2.4. Let $x$ be a random variable such that $E\{x\}>-\infty$ and $F_{1}$ $\subset F_{2} \subset \cdots$ an increasing sequence of Borel fields. Then $\lim E\left\{x \| F_{n}\right\}=y$ exists with probability one. Let $F_{\infty}$ be the smallest Borel field containing $\cup_{n} F_{n}$. Then

$$
y \geqq E\left\{x \| F_{\infty}\right\}
$$

with probability one. If $\operatorname{Pr}\{y(\omega)<\infty\}=1$, then there is equality in (2.4.1).

By Theorem 1.1, $\lim E\left\{x^{-} \| F_{n}\right\}=E\left\{x-\| F_{\infty}\right\}$ with probability one. The process $\left\{E\left\{x^{+} \| F_{n}\right\}, n \geqq 1\right\}$ is a generalized martingale and by Corollary 2.3 $\lim E\left\{x^{+} \| F_{n}\right\}=y^{+}$exists with probability one. To prove (2.4.1) it is thus sufficient to prove

$$
y^{+} \geqq E\left\{x^{+} \| F_{\infty}\right\}
$$

with probability one. Let $x^{N}=\min \left(x^{+}, N\right)$ with $N$ a positive integer. By Theorem 1.1

$$
\lim _{n \rightarrow \infty} E\left\{x^{N} \| F_{n}\right\}=E\left\{x^{N} \| F_{\infty}\right\}
$$

with probability one. Hence for each $N$ we have 


$$
y^{+} \geqq E\left\{x^{N} \| F_{\infty}\right\}
$$

with probability one. On the other hand,

$$
\lim _{N \rightarrow \infty} E\left\{x^{N} \| F_{\infty}\right\}=E\left\{x^{+} \| F_{\infty}\right\}
$$

with probability one. Hence, by (2.4.3) and (2.4.4) we have (2.4.2). Assume now that $\operatorname{Pr}\{y(\omega)<\infty\}=1$. To prove equality in (2.4.1) we need only prove that $y^{+}=E\left\{x^{+} \| F_{\infty}\right\}$ with probability one. We shall do this by proving that for any $V \in F_{\infty}$

$$
\int_{V} y^{+} d p r=\int_{V} E\left\{x^{+} \| F_{\infty}\right\} d p r
$$

The set function $\phi$ defined by $\phi(V)=\int_{V} y^{+} d p r, V \in F_{\infty}^{\prime}$, is sigma finite. Here $F_{\infty}^{\prime}$ is the Borel field of sets of $F_{\infty}$ and sets which differ from such sets by sets of probability 0 . Sigma finite means that there is a sequence $\left\{V_{j}\right\}$, $V_{j} \in F_{\infty}^{\prime}$, such that $\Omega=\cup_{j} V_{j}$ and $\phi\left(V_{j}\right)<\infty$ for all $j$. That such a sequence exists follows from the fact that $\operatorname{Pr}\left\{y^{+}(\omega)<\infty\right\}=1$. By (2.4.2) the same is true for the set function $\phi^{\prime}$ defined by $\phi^{\prime}(V)=\int_{V} E\left\{x^{+} \| F_{\infty}\right\} d p r, V \in F_{\infty}^{\prime}$. Hence, these set functions are determined by their values for $V \in \mathrm{U}_{n} F_{n}$. It is sufficient then to prove $(2.4 .5)$ for only these $V$. Let $V \in F_{m}$. By Fatou's lemma,

$$
\int_{V} y^{+} d p r \leqq \int_{V} E\left\{x^{+} \| F_{n}\right\} d p r .
$$

But, for $n>m, V \in F_{n}$ and $F_{\infty}$ so that

$$
\int_{V} E\left\{x^{+} \| F_{n}\right\} d p r=\int_{V} E\left\{x^{+} \| F_{\infty}\right\} d p r .
$$

Therefore,

$$
\int_{V} y^{+} d p r \leqq \int_{V} E\left\{x^{+} \| F_{\infty}\right\} d p r .
$$

On the other hand, by (2.4.2),

$$
\int_{V} y^{+} d p r \geqq \int_{V} E\left\{x^{+} \| F_{\infty}\right\} d p r .
$$

Hence, there must be equality in (2.4.6), as was to be proved.

3. A problem in sequential game theory. Let $Z=\left\{z_{n}, F_{n}, n \geqq 1\right\}$ be a stochastic process. In this section we shall interpret $z_{n}$ as representing a gambler's loss after $n$ plays of a gambling game, and $F_{n}$ as representing his knowledge of the past and present at that time. With this interpretation, assume that the gambler wishes to play for a while and then quit. A system 
for quitting play can be described by a random variable defined as follows:

DEFINITION 3.1. Let $\left\{F_{n}, n \geqq 1\right\}$ be an increasing sequence of Borel fields. A random variable $m$ whose values are positive integers or $+\infty$ is a stopping random variable relative to $\left\{F_{n}, n \geqq 1\right\}$ if

(a) $\{m(\omega)=j\}$ differs by at most a set of probability 0 from a set of $F_{j}$.

(b) $\operatorname{Pr}\{m(\omega)<\infty\}=1$.

The condition (a) corresponds to the fact that the decision to stop at the $j$ th play should be based only on the gambler's information at that time. Condition (b) gives the gambler a certain assurance of stopping some time. If the gambler adopts a system for quitting defined by a stopping random variable $m$, then he will have a final loss represented by $z$ with

$$
\begin{aligned}
z(\omega) & =z_{j}(\omega) & \text { if } & & m(\omega)=j \\
& =0 & \text { if } & & m(\omega)=\infty .
\end{aligned}
$$

We shall say that the pair $(m, z)$ is a stopping pair for $\left\{z_{n}, F_{n}, n \geqq 1\right\}$. We shall be interested in this section in describing a "best" stopping random variable assuming that the gambler thinks only in terms of minimizing the expected value of his final loss.

Definition 3.2. Let $Z=\left\{z_{n}, F_{n}, n \geqq 1\right\}$ be a stochastic process such that for any stopping pair $(m, z)$ the $E\{z\}$ exists. Then for $\epsilon>0,(m, z)$ is an $\epsilon$ minimizing stopping pair if for any other stopping pair $(\bar{m}, \bar{z})$ for $Z$ we have

$$
E\{\bar{z}\} \geqq E\{z\}-\epsilon .
$$

The stopping pair $(m, z)$ for $Z$ is a minimizing stopping pair if for any other stopping pair $(\bar{m}, \bar{z})$ for $Z$ we have

$$
E\{\bar{z}\} \geqq E\{z\} .
$$

It is easily seen that if $(m, z)$ is a minimizing pair for $Z$ and $(\bar{m}, \bar{z})$ any other stopping pair for $Z$, then for any $k$

$$
E\left\{\bar{z}|| F_{k}\right\} \geqq E\left\{z \| F_{k}\right\}
$$

for almost all $\omega$ of the set $\{\bar{m}(\omega) \geqq k, m(\omega) \geqq k\}$. Hence if a gambler adopts a system of stopping determined by a minimizing pair, in terms of expectation, it will always look as good as any other system. We shall, in this part, be interested in describing $\epsilon$ minimizing and minimizing pairs.

We consider first the case that $Z=\left\{z_{n}, F_{n}, n \geqq 1\right\}$ is a generalized semimartingale. The semimartingale property suggests that in this case the game is unfavorable and hence it would seem that the best thing to do is to quit as soon as possible, i.e., that a minimizing pair should be determined by $m=1$ for all $\omega$. This is not true in general. In fact Doob [3] has given examples of martingales $Z=\left\{z_{n}, F_{n}, n \geqq 1\right\}$ such that the random variables are nonnegative, $E\left\{z_{1}\right\}=1$, and $\lim _{n \rightarrow \infty} z_{n}=0$ with probability one. A martingale, we recall, is a special case of a generalized semimartingale. For such a process 
an $\epsilon$ minimizing pair exists for any $\epsilon>0$ and is determined by $\bar{m}$ defined by:

$$
\begin{aligned}
\{\bar{m}(\omega)=j\} & =\left\{z_{k}(\omega)>\epsilon, k<j, z_{j}(\omega) \leqq \epsilon\right\} \\
& =\infty \text { if undefined above. }
\end{aligned}
$$

If $(\bar{m}, \bar{z})$ is the stopping pair determined by $\bar{m}$, then $E\{\bar{z}\} \leqq \epsilon$. Hence it is obvious that in this case $m=1$ does not determine a minimizing pair.

Definition 3.3. A generalized semimartingale $\left\{z_{n}, F_{n}, n \geqq 1\right\}$ is regular if for any stopping pair $(m, z)$ the $E\{z\}$ exists and for $n=1,2, \ldots$

$$
E\left\{z \| F_{n}\right\} \geqq z_{n}
$$

for almost all $\omega$ of the set $\{m(\omega) \geqq n\}$.

It is easily seen that for $Z=\left\{z_{n}, F_{n}, n \geqq 1\right\}$ to be regular it is sufficient that (3.3) be true for all $(m, z)$ with $m \geqq k_{0}$ with $k_{0}$ a fixed integer. Hence, if $Z=\left\{z_{n}, F_{n}, n \geqq 1\right\}$ is a generalized semimartingale such that for some $k$ it is true that $Z_{k}=\left\{z_{n}, F_{n}, n \geqq k\right\}$ is regular, then $Z$ is also regular.

If $Z=\left\{z_{n}, F_{n}, n \geqq 1\right\}$ is a regular generalized semimartingale, then it is clear that a minimizing pair is determined by $m(\omega)=1$ for all $\omega$. Note that for such a process a minimizing pair for $Z_{k}=\left\{z_{n}, F_{n}, n \geqq k\right\}$ is determined by $m$ defined by $m(\omega)=k$ for all $\omega$. Hence, for a game in which the loss is represented by a regular generalized semimartingale, at any stage of the play the prospects do not warrant continuing play. We shall show that minimizing pairs for more general processes can be obtained in terms of a regular generalized semimartingale. We shall need first to prove some results concerning collections of semimartingales.

Definition 3.4. Let $\left\{x_{t}, t \in T\right\}$ be a collection of random variables. The ess $\sup _{t \in T} x_{t}$ is any random variable $x$ such that, for any $t \in T, x_{t} \leqq x$ with probability one, and if $y$ is any other random variable having this property, then $y \leqq x$ with probability one.

It is clear from the above definition that any two ess sups for a given collection are equal with probability one. Also if $T$ is denumerable, then $\sup _{t \in T} x_{t}$ is an ess sup.

Theorem 3.2. Any collection $\left\{x_{t}, t \in T\right\}$ of random variables has an ess sup.

To prove this theorem introduce a partial ordering into the set of all finitely additive set functions of bounded variation defined on the basic Borel field $B$ by putting $\phi_{1}<\phi_{2}$ if $\phi_{1}(V) \leqq \phi_{2}(V)$ for all $V \in \mathcal{B}$. Bochner and Phillips [2] have shown that any collection of such set functions which has an upper bound has a least upper bound. From their proof it is seen that if the original set functions are completely additive and absolutely continuous with respect to $\operatorname{Pr}$ measure, then the least upper bound will also have these properties. Thus if $\phi$ is the least upper bound of the set functions $\left\{\phi_{t}, t \in T\right\}$ defined by 


$$
\phi_{t}(V)=\int_{V} \arctan x_{t} d p r \quad \text { for } V \in \mathcal{B},
$$

then $\phi$ is absolutely continuous and

$$
\phi(V)=\int_{V} x d p r \quad \text { for } V \in \mathcal{B} .
$$

It is clear then that $\tan x$ is an ess sup for $\left\{x_{t}, t \in T\right\}$.

THEOREM 3.3. Let $\left\{X_{s}, s \in S\right\}$ be a collection of generalized semimartingales $X_{s}=\left\{x_{s, n}, F_{n}, n \geqq 1\right\}$ having common Borel fields. Let $x_{n}=\operatorname{ess}_{\sup _{s} \in S} x_{s, n}$. If $E\left\{x_{n}\right\}$ exists for all $n$, then $X=\left\{x_{n}, F_{n}, n \geqq 1\right\}$ is a generalized semimartingale. If the $X_{s}$ are all regular, then $X$ will also be regular provided that for any stopping pair $(m, x)$ for $X$ the $E\{x\}$ exists.

Proof. Since $x_{n}={\operatorname{ess~} \sup _{s} \in S}_{x_{s, n}}$ we have for any $s \in S$ and any $n \geqq 2$

$$
E\left\{x_{n} \| F_{n-1}\right\} \geqq E\left\{x_{8, n} \| F_{n-1}\right\}
$$

with probability one. By the semimartingale property of $X_{s}$ we have also

$$
E\left\{x_{s, n} \| F_{n-1}\right\} \geqq x_{s, n-1}
$$

with probability one. Hence for any $s \in S$ we have

$$
E\left\{x_{n} \| F_{n-1}\right\} \geqq x_{s, n-1}
$$

with probability one. Hence by the definition of ess sup

$$
E\left\{x_{n} \| F_{n-1}\right\} \geqq \underset{s \in S}{\operatorname{ess} \sup _{s, n-1}} x_{s-1}
$$

with probability one for $n \geqq 2$. Thus $X$ is a generalized semimartingale. Assume now that the $X_{s}$ are regular. Let $(m, x)$ be a stopping pair for $X$ with $m \geqq k$ with probability one. By hypothesis $E\{x\}$ exists. Then $m$ determines a stopping pair $\left(m, x^{s}\right)$ for $X_{s}$ for each $s \in S$ and by the regularity of $X_{s}$

$$
E\left\{x \| F_{k}\right\} \geqq x_{s, k}
$$

with probability one. Since $x \geqq x^{8}$ with probability one for each $s$, we have

$$
E\left\{x \| F_{k}\right\} \geqq x_{8, k} \text {. }
$$

Hence,

$$
E\left\{x \| F_{k}\right\} \geqq \underset{s \in S}{\operatorname{ess} \sup } x_{s, k}=x_{k}
$$

with probability one. As previously remarked this is sufficient to assure the regularity of $X$.

Let $X=\left\{x_{n}, F_{n}, n \geqq 1\right\}$ and $Y=\left\{y_{n}, F_{n}, n \geqq 1\right\}$ be two stochastic processes having the same Borel fields. We shall say that $Y<X$ with probability one 
if $y_{n} \leqq x_{n}$ with probability one for all $n$. We shall say that $Y=X$ with probability one if $y_{n}=x_{n}$ with probability one for all $n$.

Definition 3.4. Let $Z=\left\{z_{n}, F_{n}, n \geqq 1\right\}$ be a stochastic process. Then $X$ $=\left\{x_{n}, F_{n}, n \geqq 1\right\}$ is a maximal generalized semimartingale (maximal generalized regular semimartingale) relative to $Z$ if $X$ is a generalized semimartingale (generalized regular semimartingale) with $X<Z$ with probability one and for any other such generalized semimartingale (generalized regular semimartingale) with $Y<Z$ with probability one, we have also $Y<X$ with probability one.

It is clear from the above definition that any two maximal generalized semimartingales (maximal generalized regular semimartingales) relative to the same stochastic process are equal with probability one. Hence when we refer to "the" maximal regular generalized semimartingale we shall understand any particular representation.

In the following we shall refer to:

Hypothesis A. The stochastic process $Z=\left\{z_{n}, F_{n}, n \geqq 1\right\}$ satisfies hypothesis $\mathrm{A}$ if there exists a random variable $u$ almost everywhere nonpositive with $E\{u\}>-\infty$ and such that $z_{n} \geqq u$ with probability one for $n \geqq 1$.

THEOREM 3.4. Let $Z=\left\{z_{n}, F_{n}, n \geqq 1\right\}$ be a stochastic process satisfying hypothesis A. There exists a maximal generalized semimartingale (maximal regular generalized semimartingale) relative to $Z$.

We shall prove only the regular part of the theorem, the proof of the other half is similar. Let $u$ be the random variable of hypothesis A. By Theorem 1.2, $U:\left\{E\left\{u \| F_{n}\right\}, F_{n}, n \geqq 1\right\}$ is a martingale whose random variables are uniformly integrable. This process is then a regular generalized semimartingale (see Appendix Corollary A.1). It is clear that $U<Z$ with probability one. Let $\left\{X_{s}, s \in S\right\}$ be the class of all regular generalized semimartingales $X_{s}=\left\{x_{8, n}, F_{n}, n \geqq 1\right\}$ such that $X_{8}<Z$ with probability one. We

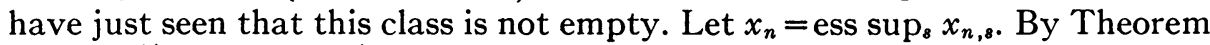
3.3, $X=\left\{x_{n}, F_{n}, n \geqq 1\right\}$ is a regular generalized semimartingale. It is clearly a maximal such process.

THEOREM 3.5. Let $Z=\left\{z_{n}, F_{n}, n \geqq 1\right\}$ be a stochastic process satisfying hypothesis $\mathrm{A}$ and let $X=\left\{x_{n}, F_{n}, n \geqq 1\right\}$ be the maximal regular generalized semimartingale (maximal generalized semimartingale) relative to $Z$. Then

$$
x_{n}=\min \left(z_{n}, E\left\{x_{n+1} \| F_{n}\right\}\right)
$$

with probability one for all $n$, and

$$
\lim _{n} \inf x_{n}=\lim _{n} \inf z_{n}
$$

with probability one. 
We prove only the regular part of the theorem, the proof of the other half being similar. If (a) is not true, then for some $r$ we have $x_{r}$ $<\min \left(z_{r}, E\left\{x_{r+1} \| F_{r}\right\}\right)$ with positive probability. Define $X^{\prime}=\left\{x_{n}^{\prime}, F_{n}, n \geqq 1\right\}$ by

$$
\begin{aligned}
x_{n}^{\prime} & =x_{n}, \\
& =\min \left(z_{r}, E\left\{x_{r+1} \| F_{r}\right\}\right), \\
& =E\left\{u \| F_{n}\right\},
\end{aligned}
$$

$$
\begin{aligned}
& n>r, \\
& n=r, \\
& n<r .
\end{aligned}
$$

Here $u$ is the random variable from hypothesis $\mathrm{A}$. Then $X^{\prime}$ is clearly a generalized semimartingale with $X^{\prime}<Z$ with probability one. Also it is regular since it has the same random variables as $X$ for $n>r$ and $X$ is regular. However, it is not true that $X^{\prime}<X$ with probability one, which is a contradiction. Hence $X$ must have property (a). We next prove (b). By hypothesis A, $E\left\{\inf _{j \geqq n} z_{j}\right\} \geqq E\{u\}>-\infty$ for all $n$. Since $\inf _{j \geqq n} z_{j}$ is monotone nondecreasing, it is easily verified that the process $\left\{E\left\{\inf _{j \geqq n} z_{j} \| F_{n}\right\}, F_{n}, n \geqq 1\right\}$ is a regular generalized semimartingale less than $Z$ with probability one. By the maximality of $X$ we have $E\left\{\inf _{j \geqq n} z_{j} \| F_{n}\right\} \leqq x_{n}$ with probability one for each $n$. By the monotonicity of the $\inf _{j \geqq n} z_{j}$ we have

$$
x_{n} \geqq E\left\{\inf _{j \geqq m} z_{j}|| F_{n}\right\},
$$$$
m \leqq n,
$$

with probability one. Therefore, for each $m$,

$$
\lim _{n} \inf x_{n} \geqq \underset{n}{\lim \inf } E\left\{\inf _{j \geqq m} z_{j} \| F_{n}\right\}
$$

with probability one. By Theorem 2.4

$$
\lim _{n} \inf E\left\{\inf _{j \geqq m} z_{j}|| F_{n}\right\} \geqq \inf _{j \geqq m} z_{j}
$$

with probability one. Hence,

$$
\lim _{n} \inf x_{n} \geqq \lim _{n} \inf z_{n}
$$

with probability one. However, since $x_{n} \leqq z_{n}$ with probability one, the last inequality must be an equality, as was to be proved.

THEOREM 3.6. Let $Z=\left\{z_{n}, F_{n}, n \geqq 1\right\}$ be a stochastic process such that hypothesis $\mathrm{A}$ is satisfied. Let $X=\left\{x_{n}, F_{n}, n \geqq 1\right\}$ be the maximal regular generalized semimartingale relative to $Z$. Then $m$ defined by

$$
\begin{aligned}
m(\omega) & =1 \text { if } x_{1}(\omega)=\infty \\
& =j \text { if } x_{k}(\omega)<z_{k}(\omega)-\epsilon \text { for } k<j \text { and } x_{j}(\omega) \geqq z_{j}(\omega)-\epsilon \\
& =\infty \text { if undefined by above }
\end{aligned}
$$

determines an $\epsilon$ minimizing pair $(m, z)$ for $Z$. For every $n$ 


$$
E\left\{x_{n}\right\}=\inf _{(\bar{m}, \bar{z})} E\{\bar{z}\}
$$

where $(\bar{m}, \bar{z})$ is any stopping pair with $\bar{m} \geqq n$ with probability one.

Proof. We shall first prove that $m$ is a stopping random variable relative to $\left\{F_{n}, n \geqq 1\right\}$. We need only prove that $\operatorname{Pr}\{m(\omega)<\infty\}=1$. Define $X^{\prime}$ $=\left\{x_{n}^{\prime}, F_{n}, n \geqq 1\right\}$ by

$$
\begin{aligned}
x_{n}^{\prime}(\omega) & =x_{n}(\omega), & & n \leqq m(\omega), \\
& =x_{m(\omega)}(\omega), & & n>m(\omega) .
\end{aligned}
$$

We shall prove that $X^{\prime}$ is a generalized martingale. Let $V \in F_{n}$. Since $x_{n}^{\prime}(\omega)$ $=x_{n+1}^{\prime}(\omega)$ if $m(\omega) \leqq n$, we have

$$
\int_{V\{m(\omega) \leqq n\}} x_{n}^{\prime} d p r=\int_{V\{m(\omega) \leqq n\}} x_{n+1}^{\prime} d p r .
$$

By Theorem 3.5(a), $x_{n}=\min \left(z_{n}, E\left\{x_{n+1} \| F_{n}\right\}\right)$ with probability one. Hence for almost all $\omega$ of the set $\{m(\omega)>n\}$ we have $x_{n}(\omega)<z_{n}(\omega)$ and $x_{n}(\omega)$ $=E\left\{x_{n+1} \| F_{n}\right\}$. Thus,

$$
\int_{V\{m(\omega)>n\}} x_{n} d p r=\int_{V\{m(\omega)>n\}} E\left\{x_{n+1} \| F_{n}\right\} d p r
$$

and since $V\{m(\omega)>n\}$ is in $F_{n}$, we have

$$
\int_{V\{m(\omega)>n\}} x_{n} d p r=\int_{V\{m(\omega)>n\}} x_{n+1} d p r .
$$

Since $x_{n}(\omega)=x_{n}^{\prime}(\omega)$ and $x_{n+1}(\omega)=x_{n+1}^{\prime}(\omega)$ for $\omega$ in the set $\{m(\omega)>n\}$, we can combine (3.6.1) and (3.6.2) to obtain

$$
\int_{V} x_{n}^{\prime} d p r=\int_{V} x_{n+1}^{\prime} d p r .
$$

Thus we have proved that $X^{\prime}$ is a generalized martingale. We shall now prove that $X^{\prime}$ satisfies the hypothesis of Corollary 2.3. Let $u$ be the random variable of hypothesis $\mathrm{A}$ and define $\boldsymbol{u}^{\prime}$ by

$$
\begin{aligned}
& u^{\prime}(\omega)=E\left\{u \| F_{k}\right\} \quad \text { if } \quad m(\omega)=k \\
& =u(\omega) \quad \text { if } \quad m(\omega)=\infty \text {. }
\end{aligned}
$$

Then $u^{\prime} \leqq 0$ with probability one and $E\left\{u^{\prime}\right\}=E\{u\}>-\infty$. We shall prove that $x_{n}^{\prime} \geqq E\left\{u^{\prime} \| F_{n}\right\}$ with probability one for all $n$. It is sufficient to prove that for $V \in F_{n}$ 


$$
\int_{V} u^{\prime} d p r \leqq \int_{V} x_{n}^{\prime} d p r .
$$

Let $k$ be an integer less than or equal to $n$. By the maximality of $X, E\left\{u \| F_{k}\right\}$ $\leqq x_{k}$ with probability one. If $m(\omega)=k$, then $u^{\prime}(\omega)=E\left\{u \| F_{k}\right\}$ and $x_{k}(\omega)$ $=x_{n}^{\prime}(\omega)$. Hence for almost all $\omega$ of the set $\{m(\omega)=k\}$ we have $u^{\prime}(\omega) \leqq x_{n}^{\prime}(\omega)$. Therefore,

$$
\int_{V\{m(\omega)=k\}} u^{\prime} d p r \leqq \int_{V\{m(\omega)=k\}} x_{n}^{\prime} d p r .
$$

Since (3.6.4) is true for any $k \leqq n$, we have

$$
\int_{V\{m(\omega) \leqq n\}} u^{\prime} d p r \leqq \int_{V(m(\omega) \leqq n\}} x_{n}^{\prime} d p r .
$$

Assume now that $k>n$. Then $u^{\prime}(\omega)=E\left\{u \| F_{k}\right\}$ on the set $\{m(\omega)=k\}$ and $V\{m(\omega)=k\} \in F_{k}$, so that

$$
\int_{V\{m(\omega)=k\}} u^{\prime} d p r=\int_{V\{m(\omega)=k\}} E\left\{u \| F_{k}\right\}=\int_{V\{m(\omega)=k\}} u d p r .
$$

If $m(\omega)=\infty$, then $u^{\prime}(\omega)=u(\omega)$, and hence

$$
\int_{V\{m(\omega)=\infty)} u^{\prime} d p r=\int_{V\{m(\omega)=\infty)} u d p r .
$$

From (3.6.6) and (3.6.7) we have

$$
\int_{V\{m(\omega)>n\}} u^{\prime} d p r=\int_{V\{m(\omega)>n\}} u d p r .
$$

Since $V\{m(\omega)>n\}$ is in $F_{n}$, the last equality gives

$$
\int_{V\{m(\omega)>n\}} u^{\prime} d p r=\int_{V\{m(\omega)>n\}} E\left\{u \| F_{n}\right\} d p r .
$$

For almost all $\omega$ of the set $\{m(\omega)>n\}$ we have $x_{n}^{\prime}(\omega)=x_{n}(\omega) \geqq E\left\{u \| F_{n}\right\}$. Hence (3.6.8) gives

$$
\int_{V\{m(\omega)>n\}} u^{\prime} d p r \leqq \int_{V\{m(\omega)>n\}} x_{n}^{\prime} d p r .
$$

From (3.6.9) and (3.6.5) we have (3.6.3) as was to be proved. Hence we can 
apply Corollary 2.3 to the $X^{\prime}$ process and we have by this corollary that $\lim x_{n}^{\prime}=x_{\infty}$ exists with probability one, $x_{\infty}(\omega)<\infty$ for almost all $\omega$ such that $x_{1}(\omega)<\infty$, and finally that

$$
E\left\{x_{\infty} \| F_{1}\right\} \leqq x_{1}^{\prime}=x_{1}
$$

with probability one. Since $x_{1}(\omega)<\infty$ for all $\omega$ of the set $\{m(\omega)=\infty\}$, we have, for almost all such $\omega, \lim x_{n}(\omega)<\infty$ and $z_{n}(\omega)>x_{n}(\omega)+\epsilon$ for all $n$. These two facts imply that for almost all $\omega$ of the set $\{m(\omega)=\infty\}$

$$
\liminf _{n} x_{n}(\omega)<\lim _{n} \inf z_{n}(\omega) .
$$

By Theorem 3.5 (b), $\operatorname{Pr}\{m(\omega)=\infty\}=0$. Thus $m$ defines a stopping random variable. Let $(m, z)$ and $(m, x)$ be the stopping pairs determined by $m$ for $Z$ and $X$ respectively. From the definition of $X^{\prime}$ we see that $x_{\infty}=x$ with probability one. By the regularity of $X$ we have

$$
E\left\{x|| F_{1}\right\} \geqq x_{1}
$$

with probability one. From (3.6.10) and (3.6.11) we have $E\left\{x \| F_{1}\right\}=x_{1}$ with probability one. From the definition of $m$,

$$
E\left\{z \| F_{1}\right\} \leqq E\left\{x \| F_{1}\right\}+\epsilon=x_{1}+\epsilon
$$

with probability one. Hence,

$$
E\{z\} \leqq E\left\{x_{1}\right\}+\epsilon .
$$

Let $\bar{m}$ be any other stopping random variable relative to $\left\{F_{n}, n \geqq 1\right\}$ and let $(\bar{m}, \bar{z})$ and $(\bar{m}, \bar{x})$ be the stopping pairs determined by $\bar{m}$ for $Z$ and $X$ respectively. Then since $X<Z$ and $X$ is regular, we have

$$
E\{\bar{z}\} \geqq E\{\bar{x}\} \geqq E\left\{x_{1}\right\} .
$$

Thus by (3.6.12) and (3.6.13) we have

$$
E\{\bar{z}\} \geqq E\{z\}-\epsilon
$$

and $(m, z)$ is an $\epsilon$ minimizing pair for $Z$. The statement (3.6) follows from the first part of the theorem if we note that the maximal generalized regular semimartingale relative to $Z_{m}=\left\{z_{n}, F_{n}, n \geqq m\right\}$ is $X_{m}=\left\{x_{n}, F_{n}, n \geqq m\right\}$.

THEOREM 3.7. Let $Z=\left\{z_{n}, F_{n}, n \geqq 1\right\}$ be a stochastic process satisfying hypothesis A. Let $X=\left\{x_{n}, F_{n}, n \geqq 1\right\}$ be the maximal regular generalized semimartingale relative to $Z$. Define $m$ by

$$
\begin{aligned}
m(\omega) & =1 \text { if } x_{1}(\omega)=\infty \\
& =j \text { if } x_{k}(\omega)<z_{k}(\omega) \text { for } k<j \text { and } x_{j}(\omega)=z_{j}(\omega) \\
& =\infty \text { if undefined by above. }
\end{aligned}
$$


If $\operatorname{Pr}\{m(\omega)<\infty\}=1$, then a minimizing pair for $Z$ exists. Such a pair can be determined by $m$. In particular this will be true if $\lim z_{n}=\infty$ with probability one. Conversely, if a minimizing pair $(\bar{m}, \bar{z})$ for $Z$ exists, then $\operatorname{Pr}\{m(\omega)<\infty\}$ $=1$ and $m$ determines a minimizing pair for $Z$ such that $m \leqq \bar{m}$ with probability one.

Assume first that $\operatorname{Pr}\{m(\omega)<\infty\}=1$. Let $(m, z)$ be the stopping pair determined by $m$. Following the proof of the first part of Theorem 3.6 we can obtain that $E\{z\}=E\left\{x_{1}\right\}$ and hence by (3.6) that $(m, z)$ is a minimizing pair. Assume now that $\lim _{n \rightarrow \infty} z_{n}=\infty$ with probability one. Again following the proof of Theorem 3.6 we can prove that $\lim x_{n}(\omega)=x_{\infty}(\omega)$ exists as a finite limit for almost all $\omega$ of the set $\{m(\omega)=\infty\}$. Thus by Theorem 3.5 (b), $\operatorname{Pr}\{m(\omega)=\infty\}=0$. To prove the converse it is sufficient to prove that if a minimizing pair $(\bar{m}, \bar{z})$ exists, then $m \leqq \bar{m}$ with probability one. If this is not true, then for some $j$ we have $V=\{\bar{m}(\omega)=j, m(\omega)>j\}$ with $\operatorname{Pr}\{V\}>0$. We shall show that this leads to a contradiction. Since by the definition of $m$ we have $x_{j}(\omega)<z_{j}(\omega)$ for $\omega \in V$, it follows that

$$
\int_{V} x_{j} d p r<\int_{V} z_{j} d p r
$$

Let $(\bar{m}, \bar{z})$ be the stopping pair for $X$ determined by $\bar{m}$. Then since $X<Z$ and (3.7.1) is true, we have

$$
E\{z\}>E\{x\} \geqq E\left\{x_{1}\right\} .
$$

The last inequality follows from the regularity of $X$. However, since $(\bar{m}, \bar{z})$ is a minimizing pair for $Z, E\{z\}=E\left\{x_{1}\right\}$ by Theorem 3.5. This contradicts (3.7.2).

Appendix. In a paper by Wald and Wolfowitz [7] it was shown that the problem of the existence of a Bayes solution in sequential decision theory could be considered, in our language, to be the problem of the existence of a minimizing pair for a suitably chosen stochastic process. These authors then proved the existence of such a stopping pair under certain hypotheses which were natural from the statistical viewpoint. Arrow, Blackwell, and Girshick [1] obtained these results for a somewhat more general case and the methods of this paper were suggested in a large part by their work. In [1] the existence of a minimizing pair is proved for a stochastic process $Z=\left\{z_{n}, F_{n}, n \geqq 1\right\}$ such that $z_{n}=r_{n}+c_{n}$ where $r_{n}$ and $c_{n}$ are non-negative, measurable with respect to $F_{n}, r_{n} \leqq K$ a fixed constant for all $n$, and $c_{n}$ is monotone nondecreasing in $n$ with $\lim _{n \rightarrow \infty} c_{n}=\infty$. As we shall see below under these hypotheses, any generalized semimartingale less than $Z$ with probability one is automatically regular. Hence by Theorem 3.6 a minimizing pair exists and is determined by the maximal generalized semimartingale relative to $Z$. Note that by Theorem 3.6 all that is needed for the existence of a minimizing pair in the case that 
the $z_{n}$ 's are non-negative is that $\lim _{n \rightarrow \infty} z_{n}=\infty$ with probability one. It is of some interest to know when the minimizing pair can be characterized by the maximal generalized semimartingale rather than the maximal regular generalized semimartingale. The following theorem is useful in this connection. The theorem is essentially the same as a theorem of Doob [3, Chapter 7]. However, we shall give the proof since the language is different and minor changes are necessary.

ThEOREM A.1. Let $\left\{x_{n}, F_{n}, n \geqq 1\right\}$ be a generalized semimartingale and $(m, x)$ be a stopping pair. Then if both

$$
\begin{gathered}
E\{x\}>-\infty, \\
\lim \inf \int_{\{m(\omega)>n\}} x_{n}^{+} d p r=0,
\end{gathered}
$$

it follows that for $n \geqq 1, E\left\{x \| F_{n}\right\} \geqq x_{n}$ for almost all $\omega$ such that $m(\omega) \geqq n$. then

Proof. It is sufficient to prove that if $V=V_{n}\{m(\omega) \geqq n\}$ where $V_{n} \in F_{n}$,

$$
\int_{V} x d p r \geqq \int_{V} x_{n} d p r
$$

If $\int_{V} x d p r=+\infty$, there is nothing to prove. Assume that $\int_{V} x d p r<\infty$. Then

$$
\begin{aligned}
\int_{V} x_{n} d p r & =\int_{V\{m(\omega)=n\}} x_{n} d p r+\int_{V\{m(\omega)>n\}} x_{n} d p r \\
& \leqq \int_{V\{m(\omega)=n\}} x_{n} d p r+\int_{V\{m(\omega)>n\}} x_{n+1} d p r .
\end{aligned}
$$

The last inequality follows from the semimartingale property of the $x_{n}$ 's. Continuing in this manner we obtain, for $k>n$,

$$
\int_{V} x_{n} d p r=\sum_{j=n}^{k} \int_{V\{m(\omega)=j\}} x_{j} d p r+\int_{V\{m(\omega)>k\}} x_{k} d p r .
$$

As $k \rightarrow \infty$ the first term on the right side of (4.1.2) approaches $\int_{V} x d p r$. By condition (b) the inequality holds in the limit.

CoRollary A.1. If $X=\left\{x_{n}, F_{n}, n \geqq 1\right\}$ is a generalized semimartingale, then $X$ is regular if either of the following conditions is satisfied:

(a) The random variables $\left\{x_{n}^{+}, n \geqq 1\right\}$ are uniformly integrable.

(b) Hypothesis A is satisfied and there exists a random variable $z \geqq 0$ with 
$E\{z\}<\infty$, and a sequence of integers $j_{1}<j_{2},<\cdots$ such that $x_{k} \geqq x_{j_{i}}-z$ for $k \geqq j_{i}$, $i \geqq 1$, for almost all $\omega$.

Either condition (a) or (b) implies that for any stopping pair $(m, z)$ conditions (a) and (b) of Theorem A.1 are satisfied. See [3, Chapter 7, p. 22] for a proof of this fact. Hence $X$ is regular.

Under the hypothesis imposed in (1) discussed in the first part of this appendix we see that hypothesis (b) of Corollary A.1 is satisfied for any generalized semimartingales less than the given process so that any such generalized semimartingale is regular.

\section{REFERENCES}

1. K. J. Arrow, D. Blackwell, and M. A. Girshick, Bayes and minimax solutions of sequential decision problems, Econometrica vol. 17 (1949).

2. S. Bochner and R. S. Phillips, Additive set functions and vector lattices, Ann. of Math. vol. 42 (1941).

3. J. L. Doob, Forthcoming book, Wiley, N. Y.

4. - Continuous parameter martingales, Proceedings of the Berkeley Symposium on mathematical statistics and probability, University of California Press, 1950.

5. H. Hahn and A. Rosenthal, Set functions, University of New Mexico Press, 1948.

6. P. Halmos, Invariants of certain stochastic transformations, Duke Math. J. vol. 5 (1939).

7. A. Wald and J. Wolfowitz, Bayes solutions of sequential decision problems, Ann. Math. Statist. vol. 21 (1950).

CoRnell University,

ITHACA, N. Y.

UNIVERSITY OF ILLINOIS,

URBANA, ILL. 